\title{
An Investigation on Engine Condition Monitoring Based on EEMD and Morphological Fractal Dimension
}

\author{
Fengli Wang \\ College of Marine Engineering, \\ Dalian Maritime University \\ Dalian 116026, China \\ wangflsky997@sina.com
}

\author{
Sihong Li \\ Technology Development Center \\ Guangdong Industry Technical College \\ Guangzhou, 510300, P. R. China
}

\author{
Yuchao Song \\ College of Marine Engineering, \\ Dalian Maritime University \\ Dalian 116026, China
}

\begin{abstract}
In respect to the nonlinear and low signal-to-noise ratio characteristics of the vibration signals measured from diesel engine, This paper conducts an investigation on diesel engine condition monitoring based on ensemble empirical mode decomposition(EEMD) and morphological fractal dimension. Firstly, the vibration signal is decomposed into a set of intrinsic mode functions(IMFs) by EEMD, and get the fault information of the characteristic IMF. Then the morphological fractal dimension of IMFs which contain diesel engine fault characteristic information is computed and as it for the characteristic parameters to identifying the diesel engine working states and fault types. The analysis of vibration signals measured from diesel engine at different states that are normal and exhaust valve leakage have been done. Results show that it can reflect nonlinear characteristics of vibration signals measured from diesel engine and monitor working condition of diesel engine accurately.
\end{abstract}

Keywords_-fractal dimension; morphology; EEMD; condition monitoring; diesel engine

\section{INTRODUCTION}

The performance and reliability of the engine is an important guarantee for the safe operation of power system. The engine valves have long been recognized as an important influence on the performance of diesel engine which can cause the change of the surface vibration response of diesel engine [1]. In consideration of the valve fault will lead to change of the surface vibration response of diesel engine, it is possible to monitor the changes in valve fault that take place with running time by analyzing the change of the surface vibration response of diesel engine.

Fractals are mathematical sets with a high degree of geometrical complexity that can model many natural phenomena [2]. This is consistent with the idea that multi-scale morphology is used to measure the geometric shape of the object being analyzed at different scales[3]. In order to use the multi-scale morphological operators to compute the fractal dimension, the improvement and optimization of this method from the angle of improving the computational efficiency, using morphological filtering operators of fractal dimension estimation[4-6]. In practice, because the measuring vibration signals mostly contain noise, the noise components have a great influence on the calculation of fractal dimension, so as to affect the accuracy of the vibration signal characteristics. The vibration signal must be denoised in order to get the morphological fractal dimension.

Ensemble empirical mode decomposition (EEMD) is able to decompose the nonlinear and non-stationary signals into a finite number of intrinsic mode components (IMF) according to the local time characteristic of the signal[7]. In this paper, the multi-scale morphological fractal dimension and EEMD are introduced into theanalysis of vibration signals.

\section{FRACTAL DIMENSION ESTIMATION BASED ON MORPHOLOGICAL OPERATIONS}

In the formalism of mathematical morphology, this cover can be obtained by using one-dimensional erosions and dilations of $f(n)$ by a function structuring element $g(m)$, the real functions of $f(n)$ and $g(m)$ are defined respectively in two discrete domains $F=\{0,1,2, \cdots N-1\}$ and $G=\{0,1,2, \cdots M-1\}$. where $f(n)$ is a time signal, $g(m)$ is a structuring element, by using one-dimensional operations erosions and dilations on $f(n)$ by a function structuring element $g(m)$, these operations are defined as

$$
f \Theta g(n)=\min _{m \in G}\{f(n+m)-g(m)\}
$$




$$
f \oplus g(n)=\min _{m \in G}\{f(n-m)+g(m)\}
$$

Hence, for one-dimensional discrete time signal $f(n)$, $n=0,1, \cdots, N$, the structuring element is defined at scales $\varepsilon$ [4],

$$
\varepsilon g(n)=g \oplus g \oplus \cdots \oplus g \quad \varepsilon \text { TIMES } .
$$

Taking $\varepsilon=1,2, \cdots, \varepsilon_{\max }$ as the range of analysis scale, $\varepsilon_{\max } \leq N / 2$, then the results of erosions and dilations of the signal $f(n)$ at different scales $\varepsilon$ are

$f \Theta \varepsilon g(n)$ and $f \oplus \varepsilon g(n)$ respectively.

The cover area of the signal at the scale $\varepsilon$ is defined

$$
A_{g}(\varepsilon)=\sum_{n=1}^{N}(f \oplus \varepsilon g(n)-f \Theta \varepsilon g(n))
$$

According to [4], $A_{g}(\varepsilon)$ satisfies the following conditions,

$$
\log \frac{A_{g}(\varepsilon)}{\varepsilon^{2}}=D_{M} \log \frac{1}{\varepsilon}+c
$$

Where $\varepsilon=1,2, \cdots, \varepsilon_{\max }$.

The Minkowski-Bouligand dimension $D_{M}$ of the signal is defined as,

$$
D_{M}=\lim _{\varepsilon \rightarrow 0} \frac{\log \left[A_{g}(\varepsilon) / \varepsilon^{2}\right]}{\log \left(\frac{1}{\varepsilon}\right)}
$$

In practice, the fractal dimension $D_{M}$ of the signal $f(n)$ is equal to the slope of a line segment fitted via least squares to the $\log \left(A_{g}(\varepsilon) / \varepsilon^{2}\right)$ and $\log \left(\frac{1}{\varepsilon}\right)$.

According to the analysis in [4], we use the flat structural element of length 3 as the unit structure element, i.e. $g(m)=\{0,0,0\}$. By using the flat structure element, the estimation result of the fractal dimension is not affected by the amplitude range of the signal and reduces the amount of calculation. According to the method of determining the maximum grid scale of periodic signal, combined with the characteristics of the diesel engine vibration signal with a working cycle, the maximum scale $\varepsilon_{\max }$ is 60 .

\section{ENSEMBLE EMPIRICAL MODE DECOMPOSITION}

\section{A. Empirical Mode Decomposition}

Eempirical mode decomposition (EMD) as an adaptive method, is developed from the simple assumption that any signal consists of different simple intrinsic modes of oscillations. With the EMD technique, any complicated signal can be decomposed into a collection of intrinsic mode functions (IMFs),, each of which must satisfy the following definition [8]: (1) In the entire data set, the number of extrema and the number of zero crossings must either be equal or differ at most by one; (2) At any point, the mean value of the envelope defined by the local maxima and the envelope defined by the local minima is zero. With the definition, any signal $\mathrm{x}(\mathrm{t})$ can be decomposed as follows:

First, find all the local extremum of the signal and use cubic spline to fit it as the local mean $\mathrm{m}(\mathrm{t})$ and extract the detail $\mathrm{h}(\mathrm{t})=$ $\mathrm{x}(\mathrm{t})-\mathrm{m}(\mathrm{t})$. Regard $\mathrm{h}(\mathrm{t})$ as new $\mathrm{x}(\mathrm{t})$ and repeat the operation above until $h(t)$ satisfies the IMF conditions, then obtain the first IMF $c_{1}(t)=h(t)$. Let the residual $r_{1}(t)=x(t)-h(t)$ be a new signal, repeat the above processes, obtain the other orders IMFs. The decomposition process can be stopped when $r_{n}(t)$ becomes a monotonic function from which no more IMF can be extracted. We finally obtain

$$
x(t)=\sum_{i=1}^{n} c_{i}(t)+r_{n}(t)
$$

Thus, we can achieve a decomposition of the signal into IMFs $c_{1}(t), c_{2}(t), \ldots, c_{n}(t)$, and a residue $r_{n}(t)$, which is the mean trend of $x(t)$. The IMFs include different frequency bands ranging from high to low. The frequency components contained in each frequency band are different and change with the variation of signal $x(t)$.

For each ${ }_{\operatorname{IMF}} C_{i}(t)$ in Eq. (1), we can always have its Hilbert transform as

$$
H\left[c_{i}(t)\right]=\frac{1}{\pi} \int_{-\infty}^{+\infty} \frac{x(\tau)}{t-\tau} d \tau
$$

With this definition, we can have an analytic signal as

$$
X_{i}(t)=c_{i}(t)+j H_{i}(\mathrm{t})=\mathrm{a}_{\mathrm{i}}(\mathrm{t}) \mathrm{e}^{j \varphi_{\mathrm{i}}(\mathrm{t})}
$$

where

$$
\begin{gathered}
a_{i}(t)=\sqrt{{c_{i}}^{2}(t)+H^{2}\left[c_{i}(t)\right]} \\
\varphi_{i}(t)=\arctan \frac{H\left[c_{i}(t)\right]}{c_{i}(t)}
\end{gathered}
$$

From Eq. (11), we can have the instantaneous frequency as

$$
\omega_{i}(t)=\frac{\varphi_{i}(t)}{2 \pi}
$$

After performing the Hilbert transform to each IMF component, the original signal can be expressed as the real part $(R P)$ in the following form

$$
x(t)=R P \sum_{i=1}^{n} a_{i}(t) e^{j \varphi_{i}(t)}
$$




\section{B. Ensemble Empirical Mode Decomposition}

EMD is based on the local characteristic time scales of a signal and could decompose the complicated signal function (IMF). The IMFs represent the natural oscillatory mode embedded in the signal and work as the basis functions, which are determined by the signal itself, rather than pre-determined kernels.

However, one of the major drawbacks of EMD is the mode mixing problem. To alleviate the problem of mode mixing in EMD, ensemble empirical mode decomposition (EEMD) is proposed, which is a noise-assisted data analysis method and defines the true IMF components as the mean of an ensemble of trials[7]. The EEMD algorithm can be given as follows.

(1) Add a white noise series to the targeted data.

$$
X(t)=x(t)+N(t)
$$

(2) Decompose the data with added white noise into IMFs.

$$
X(t)=\sum_{j=1}^{n} c_{j}(t)+r_{n}(t)
$$

(3) Repeat step 1 and step 2 again and again, but with different white noise series each time, $i=1 \sim m$,

$$
\begin{gathered}
X_{i}(t)=x(t)+N_{i}(t) \\
X_{i}(t)=\sum_{j=1}^{n} c_{i j}(t)+r_{i n}(t)
\end{gathered}
$$

(4) Obtain the means of corresponding IMFs of the decompositions as the final result.

$$
c_{j}(t)=\frac{1}{m} \sum_{i=1}^{m} c_{i j}(t)
$$

\section{Simulation Analysis}

In order to verify the effectiveness of noise signal decomposed by using EEMD, a simulation signal is decomposed by using EMD and EEMD, which is composed of a low frequency sine and a small impact component, Fig. 1 shows the time domain waveform of simulated signal. Fig. 2 shows the decomposition results by EMD. Because of abnormal interference of noise, LWD generates the mode mixture, and lead to the pseudo IMFs which cannot satisfy the requirement of feature extraction. Sinusoidal signal and impact signal is decomposed into the IMF component $\mathrm{C} 1$; moreover, the sinusoidal signal is decomposed into two IMF components C1 and C2, the decomposition results produced a serious distortion, EMD generates the mode mixture, and lead to the pseudo IMFs which cannot satisfy the requirement of feature extraction.

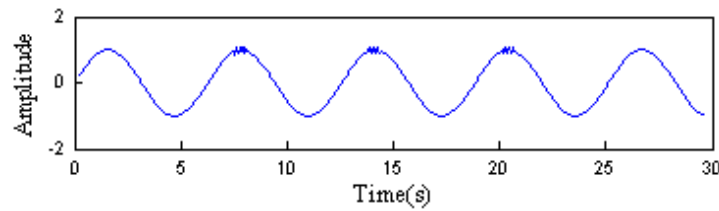

Fig. 1. Time domain waveform of simulated signal

The simulation signal is decomposed by EEMD, the average number of decomposition is 100 , and the amplitude of noise is 0.01 times of the standard deviation of the signal._The decomposition results in Fig. 3 shows, C1 component corresponding impact signal components, components corresponding to C2 sine signal and sine signal, the small impact component and the sine signal is accurately decomposed.

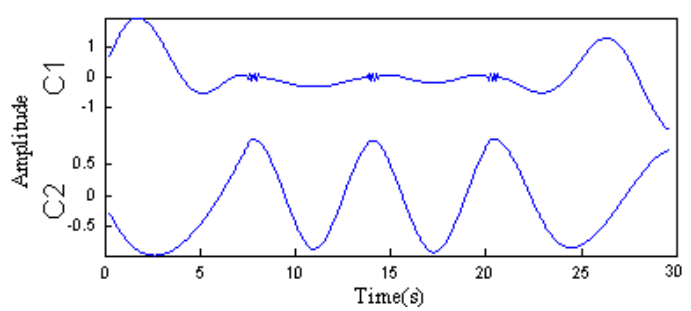

Fig. 2. Decomposition results by EMD

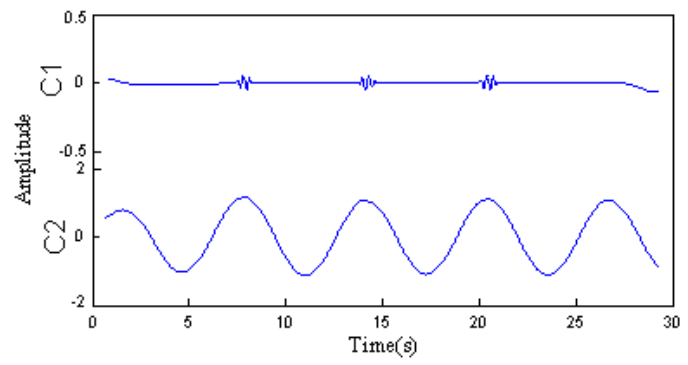

Fig. 3. Decomposition results by EEMD

\section{ExhaUst VALVE LEAKAGE MONITORING}

As one of the important parts of a diesel engine, the exhaust valve easily suffers from leakage under the alternating load. The proposed method is applied to diagnosing the Exhaust valve leakage of diesel engine. In a certain type of four cylinder six stroke diesel engine under normal operating conditions and varying degrees of exhaust valve leakage under the three conditions. We collected the vibration signals from the same cylinder, which represent the engine in normal state, slightly leakage, and serious leakage condition. All data were sampled at $25.6 \mathrm{kHz}$, and the analyzing frequency is $10 \mathrm{kHz}$. The rotating speed of the diesel engine is $1100 \mathrm{r} / \mathrm{min}$ around. Fig.4 a) c) show the vibration signals of the engine exhaust valve in the three conditions.

The fractal dimensions of the vibration signal are computed directly by using the fractal dimension estimation algorithm based on the morphology, as shown in Fig. 5. Because under the three conditions the response in the background noise are similar in morphology, so the fractal dimensions are very close, it is difficult to distinguish the exhaust valve of the state.

EEMD is used to decompose the vibration response of the diesel engine from high frequency to low frequency, and it can be decomposed into several IMF components, which represent the different frequency components of the original vibration signal. The mechanism from the exhaust valve leakage fault, natural vibration component contains the fault information belonging to the high frequency components, so the fractal dimension is only IMF1 component calculation decomposed, can quantify the working state of the exhaust valve. Therefore, 
morphological fractal dimension is used to estimate the IMF1 of diesel engine vibration response under the three conditions, the results are shown in Fig. 6.The fractal dimension can be clearly separated from the three district conditions of diesel engine exhaust valve leakage.

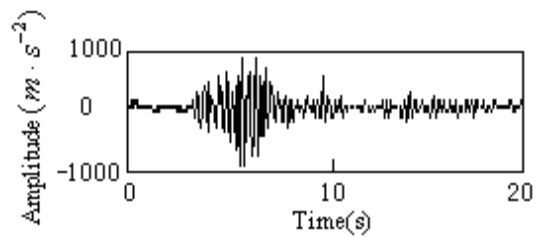

(a) Normal state

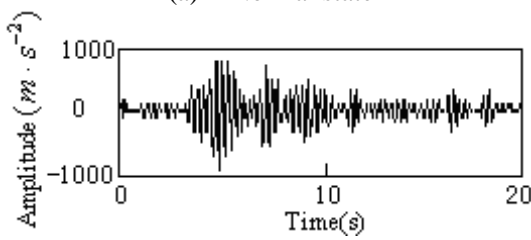

(b) Slightly leakage

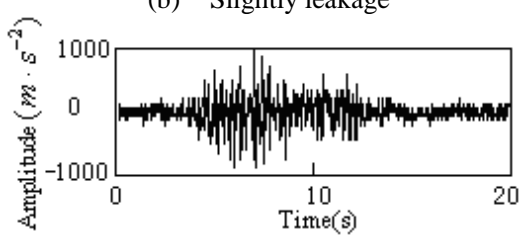

(c) Serious leakage

Fig. 4. Vibration signals of diesel engine for valve leakage states

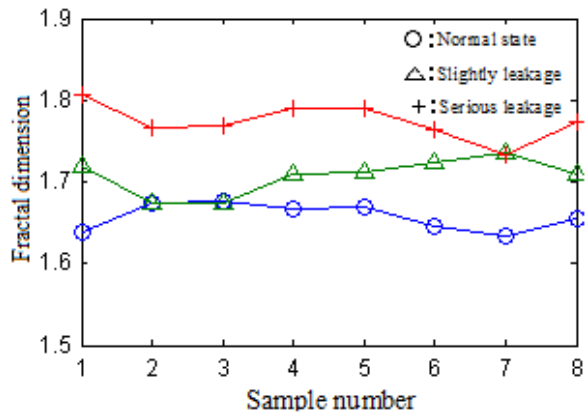

Fig. 5. Morphological fractal dimension of vibration signals under the three conditions

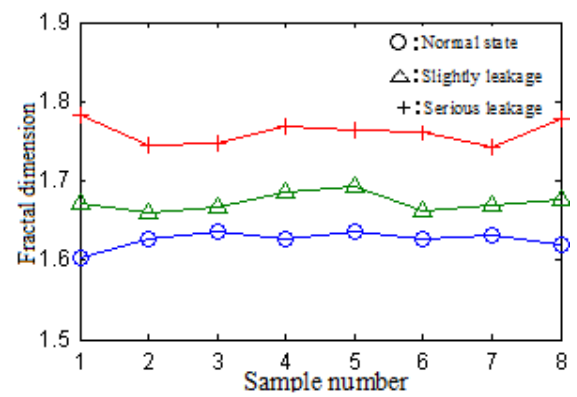

Fig. 6. Morphological fractal dimension of the characteristic IMF of vibration signals under the three conditions

\section{CONCLUSIONS}

EEMD can effectively separate the characteristic components from the nonlinear non-stationary vibration signal and the noise measured from the diesel engine, thereby improving the signal to noise ratio of the signal fractal analysis. By morphological fractal dimension analysis of the IMF component which contains the fault feature, morphological fractal dimension can quantitatively describe the geometric characteristics of the vibration signal of diesel engine. Through the analysis of the fractal dimension of the vibration signals measured from diesel engine, the results show that the proposed method can be applied to extract the fault characteristic information of the surface vibration signal and monitor the working conditions effectively.

\section{ACKNOWLEDGMENT}

This research was financially supported by the Science Research Project of Liaoning Provincial Department of Education(L2015069), the National Science Foundation (11272093) and the Opening Project of Technology Development Center for Polymer Processing Engineering of Guangdong Province, Guangdong Industry Technical College (201503).

\section{REFERENCES}

[1] Z. Geng, J. Chen, J. B. Hull, "Analysis of engine vibration and design of an applicable diagnosing approach,” International Journal of Mechanical Sciences. 45,2003, pp.1391-1410.

[2] B. B. Mandelbrot, "The fractal geomety of nature," New York: Freeman, 1982.

[3] L J Zhang, J W Xu, J H Yang, et al. "Multiscale morphology analysis and its application to fault diagnosis," Mechanical Systems and Signal Processing, 22,2008, pp.597-610.

[4] P. Maragos, F K Sun, "Measuring the fractal dimension of signals: morphological covers and iterative optimization,” Ieee Transactions On Signal Processing, 41,1993, pp.108-121.

[5] P Maragos, "Pattern spectrum and multiscale shape representation," Transactions on Pattern Analysis and Machine Intelligence, , 11(7), 1989, pp.701-716.

[6] P Maragos, "Pattern spectrum of images and morphological shape-size complexity,” IEEE International Conference on ICASSP, Dallas, 1987, pp.241-244.

[7] Z H Wu, N E Huang, "Ensemble empirical mode decomposition: a noise assisted data analysis method,” Advances in Adaptive Data Analysis, 1,2009, pp.1-41.

[8] N. E. Huang, Z Shen, S R Long, et al, "The Empirical Mode Decomposition and The Hilbert Spectrum for Nonlinear Nonstationary Time Series Analysis,” Proceedings of the Royal Society of London, Vol.454,1998, pp.903-995. 